\title{
T MEASURE OF CARTESIAN PRODUCT SETS. II
}

\author{
BY
}

\author{
LAWRENCE R. ERNST( $\left.{ }^{(}\right)$
}

\begin{abstract}
It is proven that there exists a subset $A$ of Euclidean 2space such that the 2-dimensional $T$ measure of the Cartesian product of an interval of unit length and $A$ is less than the 1-dimensional $T$ measure of $A$. In a previous paper it was shown that there exists a subset of Euclidean 2-space such that the reverse inequality holds. $T$ measure is the first measure of its type for which it has been shown that both of these relations are possible.
\end{abstract}

1. Introduction. There are many 1-dimensional measures and 2-dimensional measures over Euclidean $n$-space, $\mathbf{R}^{n}$, which generalize the concept of length and area respectively. These measures were studied extensively by $\mathrm{H}$. Federer [6]. One question concerning them is whether for any of these measures there exists in $\mathbf{R}^{\mathbf{3}}$ both product sets for which area exceeds the product of (finite) length by (finite) length and other product sets for which the opposite is true. This question had until now not been answered. In fact, although examples were constructed showing for Hausdorff measure that area may be greater than the product of length by length [1], [7] and for Carathéodory measure that area may be less than the product of length by length [8], it was also proven that for both of these measures the respective reverse relations cannot hold [5], [9].

The main purpose of this paper is to provide an answer by means of $T$ measure, which we do by constructing a subset $A$ of $\mathbf{R}^{2}$ that we prove satisfies the relation $T^{2}([0,1] \times A)<T^{1}(A)$ (Theorem 5.1). Previously the author [4] gave an example for which the opposite inequality holds.

As a corollary to our principal result we obtain that the 2-dimensional Carathéodory measure of $[0,1] \times A$ is less than the 1-dimensional Carathéodory measure of $A$ (Corollary 5.2). We also deduce that the 2-dimensional $T$ measure of $[0,1] \times A$ is less than its 2-dimensional Hausdorff measure (Corollary 5.3), thus showing that these two measures are distinct. Both of these corollaries are new proofs of previously known results. The former, as mentioned above, was first proven by G. Freilich [8], while the latter was first obtained by the author [3].

Received by the editors March 27, 1975.

AMS (MOS) subject classifications (1970). Primary 28A35, $28 \mathrm{A75}$.

Key words and phrases. 1-dimensional measures, 2-dimensional measures, Cartesian product sets, $T$ measure, Hausdorff measure, Carathéodory measure.

(1) Research supported by a grant from the City University of New York Faculty Research Award Program. 
2. Preliminaries. In general we adopt the notation and terminology of [6]. Presented in this section are some additional definitions that we use.

Define $r_{1}(x, y)=x$ and $r_{2}(x, y)=y$ for $x, y \in \mathbf{R}$.

For $a, b \in \mathbf{R}$ let $[a, b]=\{x: a \leqslant x \leqslant b\}$.

For $\varnothing \neq S \subset \mathbf{R}^{n}$ let

and

$$
t^{1}(S)=\operatorname{diam} S
$$

$$
t^{2}(S)=(\pi / 4) \sup \left\{\left|\left(a_{1}-b_{1}\right) \wedge\left(a_{2}-b_{2}\right)\right|: a_{1}, b_{1}, a_{2}, b_{2} \in S\right\} .
$$

These are the gauge functions used in defining $T^{1}$ and $T^{2}$ respectively $[6,2.10 .3]$.

The following series of definitions culminate in the definition of the set $A$ referred to in the introduction. First for $\varnothing=S \subset \mathbf{R}^{2}, a, b \in \mathbf{R}$ let

$$
\begin{aligned}
\Phi(S, a, b)= & {\left[\inf r_{1}(S)+a \operatorname{diam} r_{1}(S), \inf r_{1}(S)+\left(a+10^{-24}\right) \operatorname{diam} r_{1}(S)\right] } \\
& \times\left[\inf r_{2}(S)+b \operatorname{diam} r_{2}(S), \inf r_{2}(S)+\left(b+10^{-24}\right) \operatorname{diam} r_{2}(S)\right]
\end{aligned}
$$

Next let $\Gamma=\left\{.5\left(1-10^{-11}\right), .5\left(1+10^{-11}\right)-10^{-24}\right\}$. Then inductively define the four sequences $F_{0}, F_{1}, F_{2}, \ldots, G_{1}, G_{2}, G_{3}, \ldots, H_{1}, H_{2}, H_{3}, \ldots$, $K_{1}, K_{2}, K_{3}, \ldots$ by taking $F_{0}=[-.5, .5] \times[-.5, .5]$ and, for $j \geqslant 1$, letting

$$
\begin{aligned}
& G_{j}=\left\{\Phi\left[S, 2(i-1) 10^{24}, k\right]: S \in F_{j-1}, i=1,2, \ldots, 2.4975 \cdot 10^{23}, k \in \Gamma\right\}, \\
& \begin{aligned}
& H_{j}=\left\{\Phi\left[S, .5005+(2 i-1) 10^{-24}, k\right]:\right. S \in F_{j-1}, \\
&\left.i=1,2, \ldots, 2.4975 \cdot 10^{23}, k \in \Gamma\right\}, \\
& K_{j}=\left\{\Phi\left[S, k, .5+2(i-1) 10^{-22}\right]: s \in F_{j-1},\right. \\
&\left.i=1,2, \ldots, 5 \cdot 10^{20}, k \in \Gamma\right\}, \\
& F_{j}=G_{j} \cup H_{j} \cup K_{j} .
\end{aligned}
\end{aligned}
$$

Finally let $A=\bigcap_{j=0}^{\infty} \cup F_{j}$ and abbreviate $[0,1] \times A$ by $E$.

3. The 1-dimensional $T$ measure of $A$.

3.1. Lemma. If $D \subset A, \operatorname{diam} D>0$ and $k=\sup \{i: D \subset S$ for some $S \in$ $\left.F_{i}\right\}+1$, then

$$
t^{1}(D) \geqslant\left[\operatorname{card}\left(F_{k} \cap\{S: S \cap D \neq \varnothing\}\right)\right] 10^{-24 k} .
$$

Proof. Let $c$ denote the center of the element of $F_{k-1}$ containing $D$, $B=\left\{10^{24(k-1)}(x-c): x \in D\right\}$ and $n=\operatorname{card}\left(F_{1} \cap\{S: S \cap B \neq \varnothing\}\right)$. Then clearly $t^{1}(B)=10^{24(k-1)} t^{1}(D), n=\operatorname{card}\left(F_{k} \cap\{S: S \cap D \neq \varnothing\}\right)$. Consequently, to obtain our conclusion it suffices to prove that

$$
t^{1}(B) \geqslant n 10^{-24} \text {. }
$$


To deduce (1) we first let $X_{1}=G_{1} \cap\{S: S \cap B \neq \varnothing\}, X_{2}=H_{1} \cap\{S:$ $S \cap B \neq \varnothing\}, X_{3}=K_{1} \cap\{S: S \cap B \neq \varnothing\}, m_{1}=$ card $r_{1}\left(X_{1}\right), m_{2}=$ card $r_{1}\left(X_{2}\right)$, $m_{3}=$ card $r_{2}\left(X_{3}\right)$, and $x_{i}=\operatorname{card} X_{i}$ for $i=1,2,3$. We then divide the proof of (1) into eight cases.

Case I. $X_{1}=X_{2}=\varnothing$ and $n \geqslant 3$. It follows from the definition of $K_{1}$ and the inequality $2 m_{3} \geqslant n$ that

$$
t^{1}(B) \geqslant t^{1}\left[r_{2}(B)\right] \geqslant 2\left(m_{3}-1\right) 10^{-22}-10^{-24} \geqslant n 10^{-24} .
$$

Case II. $X_{1}=X_{2}=\varnothing$ and $n=2$. Let $S, T$ denote the two elements of $X_{3}$. Then

$$
t^{1}(B) \geqslant \operatorname{dist}(S, T) \geqslant 2 \cdot 10^{-22}-10^{-24} \geqslant n 10^{-24} .
$$

Case III. $X_{1} \neq \varnothing, X_{2} \neq \varnothing$ and $2\left(m_{1}+m_{2}-1\right) \geqslant x_{1}+x_{2}$. We deduce from the definitions of $G_{1}, H_{1}$ and the inequalities $2\left(m_{1}+m_{2}-1\right) \geqslant x_{1}+x_{2}$, $x_{1}+x_{2} \geqslant n-10^{21}$ that

$$
t^{1}(B) \geqslant t^{1}\left[r_{1}(B)\right] \geqslant 2\left(m_{1}+m_{2}-1\right) 10^{-24}+10^{-3} \geqslant n 10^{-24} .
$$

Case IV. $X_{1} \neq \varnothing, X_{2} \neq \varnothing$ and $2 m_{1}+2 m_{2}-1 \leqslant x_{1}+x_{2}$. There then exist $S \in X_{1}, T \in X_{2}$ such that

$$
\begin{aligned}
& \operatorname{dist}\left[r_{1}(S), r_{1}(T)\right] \geqslant 2\left(m_{1}+m_{2}-1\right) 10^{-24}+10^{-3}, \\
& \operatorname{dist}\left[r_{2}(S), r_{2}(T)\right]=10^{-11}-2 \cdot 10^{-24} .
\end{aligned}
$$

Combining the former relation with the inequalities $2 m_{1} \geqslant x_{1}, 2 m_{2} \geqslant x_{2}, x_{1}+$ $x_{2} \geqslant n-10^{21}$ and $n \leqslant 10^{24}$, we then find that

$$
\begin{aligned}
\left(\operatorname{dist}\left[r_{1}(S), r_{1}(T)\right]\right)^{2} & \geqslant\left[(n-2) 10^{-24}\right]^{2} \geqslant\left(n^{2}-4 n\right) 10^{-48} \\
& \geqslant n^{2} 10^{-48}-4 \cdot 10^{-24} .
\end{aligned}
$$

Consequently

$$
\begin{aligned}
t^{1}(B) & \geqslant \operatorname{dist}(S, T)=\left[\left(\operatorname{dist}\left[r_{1}(S), r_{1}(T)\right]\right)^{2}+\left(\operatorname{dist}\left[r_{2}(S), r_{2}(T)\right]\right)^{2}\right]^{1 / 2} \\
& \geqslant n 10^{-24} .
\end{aligned}
$$

Case V. $X_{2}=X_{3}=\varnothing$ and $2 m_{1}-3 \geqslant n$. We deduce from the definition of $G_{1}$ that

$$
t^{1}(B) \geqslant t^{1}\left[r_{1}(B)\right] \geqslant\left(2 m_{1}-3\right) 10^{-24} \geqslant n 10^{-24} .
$$

Case VI. $X_{2}=X_{3}=\varnothing$ and $2 m_{1}-2 \leqslant n$. Let

$$
C=\left\{\{Y, Z\}: Y, Z \in X_{1}, Y \neq Z \text { and } r_{1}(Y)=r_{1}(Z)\right\} \text {. }
$$

Noting that 


$$
\text { card } C=n-m_{1} \geqslant n-[(n+2) / 2]=(n-2) / 2,
$$

we then use this relation, the definition of $G_{1}$ and the inequality $n \leqslant 10^{24}$ to find that there exist $S, T \in \cup C$ satisfying

$$
\begin{aligned}
\left(\operatorname{dist}\left[r_{1}(S), r_{1}(T)\right]\right)^{2} & \geqslant\left[(2 \operatorname{card} C-3) 10^{-24}\right]^{2} \geqslant(n-5)^{2} 10^{-48} \\
& \geqslant\left(n^{2}-10 n\right) 10^{-48} \geqslant n^{2} 10^{-48}-10^{-23}, \\
\operatorname{dist}\left[r_{2}(S), r_{2}(T)\right] & =10^{-11}-2 \cdot 10^{-24} .
\end{aligned}
$$

Consequently $t^{1}(B) \geqslant \operatorname{dist}(S, T) \geqslant n 10^{-24}$.

Case VII. $X_{1} \neq \varnothing, X_{2}=\varnothing$ and $X_{3} \neq \varnothing$. It follows from the definition of $G_{1}$ and the inequality $2 m_{1} \geqslant x_{1}$ that there exists $S \in X_{1}$ satisfying

$$
\begin{aligned}
\operatorname{dist}\left[r_{1}(S), 0\right] & \geqslant\left(2 m_{1}-1\right) 10^{-24}+5 \cdot 10^{-4} \\
& \geqslant\left(x_{1}-1\right) 10^{-24}+5 \cdot 10^{-4} .
\end{aligned}
$$

Similarly from the definition of $K_{1}$ and the inequality $2 m_{3} \geqslant x_{3}$ it follows that there exists $T \in X_{3}$ satisfying

$$
\operatorname{dist}\left[r_{2}(T), 0\right] \geqslant 2\left(m_{3}-1\right) 10^{-22} \geqslant\left(x_{3}-2\right) 10^{-22} \text {. }
$$

From (2) we obtain

(4) $\operatorname{dist}\left[r_{1}(S), r_{1}(T)\right] \geqslant \operatorname{dist}\left[r_{1}(S), 0\right]-10^{-11} / 2 \geqslant x_{1} 10^{-24}+4 \cdot 10^{-4}$.

Since (1) immediately follows from (4) otherwise, we may assume that $x_{1} \leqslant n-$ $4 \cdot 10^{20}$. Then $x_{3}=n-x_{1} \geqslant 4 \cdot 10^{20}$, which we combine with (3) to deduce

$$
\operatorname{dist}\left[r_{2}(S), r_{2}(T)\right] \geqslant \operatorname{dist}\left[r_{2}(T), 0\right]-10^{-11} / 2 \geqslant 3.9 \cdot 10^{-2} \text {. }
$$

We next use the inequalities (4), $x_{1} \geqslant n-10^{21}$ and $n \leqslant 10^{24}$ to obtain

$$
\begin{aligned}
\left(\operatorname{dist}\left[r_{1}(S), r_{1}(T)\right]\right)^{2} & \geqslant\left[\left(n-10^{21}\right) 10^{-24}+4 \cdot 10^{-4}\right]^{2} \\
& =\left(n 10^{-24}-6 \cdot 10^{-4}\right)^{2} \geqslant n^{2} 10^{-48}-12 n 10^{-28} \\
& \geqslant n^{2} 10^{-48}-12 \cdot 10^{-4} .
\end{aligned}
$$

Finally (5) and (6) yield $t^{1}(B) \geqslant \operatorname{dist}(S, T) \geqslant n 10^{-24}$.

Case VIII. $X_{1}=\varnothing$ and $X_{2} \neq \varnothing$. Because of the symmetry between $G_{1}$ and $H_{1}$, it immediately follows from Cases V, VI and VII that $t^{1}(B) \geqslant n 10^{-24}$.

3.2. THEOREM. $T^{1}(A) \geqslant 1$.

Proof. Consider any countable covering of $A$ consisting of nonempty subsets of $A$ that are open in $A$, and let $W$ be a finite subcover. For $T \in W$ define $\zeta(T)=\sup \left\{i: T \subset S\right.$ for some $\left.S \in F_{i}\right\}+1$ and let $z=\sup \{\zeta(T): T \in W\}$. Then clearly for each $T \in W$ we have 


$$
\begin{aligned}
{\left[\operatorname { c a r d } \left(F_{\zeta(T)} \cap\right.\right.} & \{S: S \cap T \neq \varnothing\})] 10^{-24 \zeta(T)} \\
& \geqslant\left[\operatorname{card}\left(F_{z} \cap\{S: S \cap T \neq \varnothing\}\right)\right] 10^{-24 z},
\end{aligned}
$$

which we combine with Lemma 3.1 to obtain

$$
\sum_{T \in W} t^{1}(T) \geqslant \sum_{T \in W}\left[\operatorname{card}\left(F_{z} \cap\{S: S \cap T \neq \varnothing\}\right)\right] 10^{-24 z} .
$$

Furthermore, since $W$ covers $A$, and $S \cap A \neq \varnothing$ for any $S \in F_{z}$, it follows that

$$
\sum_{T \in W} \operatorname{card}\left(F_{z} \cap\{S: S \cap T \neq \varnothing\}\right) \geqslant 10^{24 z} \text {. }
$$

The last two results yield $\Sigma_{T \in W} t^{1}(T) \geqslant 1$; hence $T^{1}(A) \geqslant 1$.

4. The 2-dimensional $T$ measure of $E$.

4.1. Definitions. Define

$$
p_{1}(x,(y, z))=x, \quad p_{2}(x,(y, z))=y, \quad p_{3}(x,(y, z))=z, \quad q(x,(y, z))=(y, z)
$$

for $(x,(y, z)) \in \mathbf{R} \times \mathbf{R}^{2}$.

For $i=1,2, \ldots,\left(10^{24}-10^{21}\right) / 4$ let

$$
\begin{gathered}
R_{i}=\left\{S \cap A: S \in G_{1} \cup H_{1} \text { and } \operatorname{diam}\left[r_{1}(S) \cup\{0\}\right]=2 i 10^{-24}+5 \cdot 10^{-4}\right\}, \\
\lambda(i)=\left[1-\left(4 i 10^{-24}+10^{-3}\right)^{2}\right]^{1 / 2} .
\end{gathered}
$$

Then for $h>0$ detine

$$
\begin{aligned}
M(h)= & \bigcup_{i=1}^{\left(10^{24}-10^{21}\right) / 4}\left[\{x:-.5 \lambda(i) h \leqslant x<.5 \lambda(i) h\} \times \bigcup R_{i}\right] \\
& \cup\left(\{x:-.5 h \leqslant x<.5 h\} \times\left[\left(\bigcup K_{1}\right) \cap A\right]\right) .
\end{aligned}
$$

4.2. LEMMA. If $h>0$ then $T^{2}[M(h)] \geqslant(\pi / 4)\left(1+2 \cdot 10^{-10}\right) h T^{2}(E)$.

Proof. Since card $R_{i}=4$ for each $i$, it follows that

$$
T^{2}\left[\{x:-.5 \lambda(i) h \leqslant x<.5 \lambda(i) h\} \times \bigcup R_{i}\right]=\lambda(i) h 4 \cdot 10^{-24} T^{2}(E),
$$

which then yields

$$
\begin{aligned}
T^{2}\left(q^{-1}\left[\cup\left(G_{1} \cup H_{1}\right)\right] \cap M(h)\right) \\
={ }^{\left(10^{24}-10^{21}\right) / 4} \lambda(i) 4 \cdot 10^{-24} h T^{2}(E) .
\end{aligned}
$$

Next we deduce from the definition of the Riemann integral that

$$
\left(10^{24}-10^{21}\right) / 4
$$


which we combine with (7) to obtain

$$
\begin{aligned}
T^{2}\left(q^{-1}\left[\cup\left(G_{1} \cup H_{1}\right)\right] \cap M(h)\right) \\
\qquad\left[\int_{10^{-3}}^{1}\left(1-x^{2}\right)^{1 / 2} d x-4 \cdot 10^{-24}\right] h T^{2}(E) \\
\geqslant\left(\pi / 4-9.9999984 \cdot 10^{-4}\right) h T^{2}(E) .
\end{aligned}
$$

Furthermore, since card $K_{1}=10^{21}$ we have

$$
T^{2}\left[q^{-1}\left(U K_{1}\right) \cap M(h)\right]=10^{-3} h T^{2}(E) .
$$

Finally, to complete the proof we combine (8) and (9).

4.3. LEMMA. If $h \geqslant 10^{5}$ then $t^{2}[M(h)] \leqslant(\pi / 4)\left(1+4 \cdot 10^{-11}\right) h$.

Proof. Let

$$
\begin{aligned}
& C=\left\{(x,(y, 0)):(x,(y, z)) \in q^{-1}\left[\bigcup\left(G_{1} \cup H_{1}\right)\right] \cap M(h) \text { for some } z \in \mathbf{R}\right\}, \\
& D=\left\{(x,(0, z)):(x,(y, z)) \in q^{-1}\left(\bigcup K_{1}\right) \cap M(h) \text { for some } y \in \mathbf{R}\right\}
\end{aligned}
$$

For $S, T \subset \mathbf{R} \times \mathbf{R}^{2}$ define $S-T=\{a-b: a \in S, b \in T\}$. We note that if $a \in M(h)$ then there exists $b \in C \cup D$ for which $|a-b| \leqslant 10^{-11} / 2$. Consequently, corresponding to any $u_{1}, u_{2} \in M(h)-M(h)$ there exist $v_{1}, v_{2} \in(C \cup D)-$ $(C \cup D)$ satisfying $\left|u_{1}-v_{1}\right| \leqslant 10^{-11}$ and $\left|u_{2}-v_{2}\right| \leqslant 10^{-11}$, which together with the inequality $h \geqslant 10^{5}$ yield

$$
\begin{aligned}
\left|u_{1} \wedge u_{2}\right|= & \left|\left[v_{1}+\left(u_{1}-v_{1}\right)\right] \wedge\left[v_{2}+\left(u_{2}-v_{2}\right)\right]\right| \\
\leqslant & \left|v_{1} \wedge v_{2}\right|+\left|v_{1}\right| \cdot\left|u_{2}-v_{2}\right| \\
& +\left|v_{2}\right| \cdot\left|u_{1}-v_{1}\right|+\left|u_{1}-v_{1}\right| \cdot\left|u_{2}-v_{2}\right| \\
& \leqslant\left|v_{1} \wedge v_{2}\right|+3 \cdot 10^{-11} h .
\end{aligned}
$$

To obtain our conclusion it therefore suffices to show that

(10) $\left|v_{1} \wedge v_{2}\right| \leqslant\left(1+10^{-11}\right) h$ for every $v_{1}, v_{2} \in(C \cup D)-(C \cup D)$.

To establish (10) we first let $p_{i}\left(v_{1}\right)=a_{i}$ and $p_{i}\left(v_{2}\right)=b_{i}$ for $i=1,2,3$, and note that

(11) $\left|v_{1} \wedge v_{2}\right|=\left[\left(a_{1} b_{2}-a_{2} b_{1}\right)^{2}+\left(a_{1} b_{3}-a_{3} b_{1}\right)^{2}+\left(a_{2} b_{3}-a_{3} b_{2}\right)^{2}\right]^{1 / 2}$.

We then divide the proof of (10) into five cases. 
Case I. $v_{1} \in C-C$. Let $e_{1}=(1,(0,0)), e_{2}=(0,(1,0))$. We observe that definitions of $M(h), C$ imply

$$
\left|\left(a_{1} / h\right) e_{1}+a_{2} e_{2}\right| \leqslant 1,
$$

which in turn yields

$$
\left|a_{1}\right| \leqslant\left(1-a_{2}^{2}\right)^{1 / 2} h
$$

We then divide Case I into three subcases.

Case I.A. $v_{2} \in C-C$. Noting that $\left|\left(b_{1} / h\right) e_{1}+b_{2} e_{2}\right| \leqslant 1$ and combining this with the relations (11), $a_{3}=b_{3}=0$ and (12), we obtain

$$
\begin{aligned}
\left|v_{1} \wedge v_{2}\right| & =\left|a_{1} b_{2}-a_{2} b_{1}\right| \\
& =\left|\left[\left(a_{1} / h\right) e_{1}+a_{2} e_{2}\right] \wedge\left[\left(b_{1} / h\right) e_{1}+b_{2} e_{2}\right]\right| h \leqslant h .
\end{aligned}
$$

Case I.B. $v_{2} \in D-D$. We deduce from the relations (11), $b_{2}=a_{3}=0$, $\left|b_{1}\right| \leqslant h,(13),\left|b_{3}\right| \leqslant .1,\left|a_{2}\right| \leqslant 1$ and $h \geqslant 10^{5}$ that

$$
\begin{aligned}
\left|v_{1} \wedge v_{2}\right| & =\left[\left(a_{2} b_{1}\right)^{2}+\left(a_{1} b_{3}\right)^{2}+\left(a_{2} b_{3}\right)^{2}\right]^{1 / 2} \\
& \leqslant\left[a_{2}^{2} h^{2}+.01\left(1-a_{2}^{2}\right) h^{2}+.01\right]^{1 / 2} \\
& \leqslant\left(h^{2}+.01\right)^{1 / 2} \leqslant\left(1+10^{-11}\right) h .
\end{aligned}
$$

Case I.C. $v_{2} \in C-D$. Choose $x \in C, y \in D$ satisfying $v_{2}=x-y$. Then from the definitions of $M(h), C$ we obtain $\left|p_{1}(x)\right| \leqslant .5\left(1-4 b_{2}^{2}\right)^{1 / 2} h$, which implies that $\left|\left[p_{1}(x) / h\right] e_{1}+b_{2} e_{2}\right| \leqslant .5$. Furthermore $\left|p_{1}(y)\right| \leqslant .5 h$. The last two inequalities together with the identity $b_{1}=p_{1}(x)-p_{1}(y)$ and (12) yield

$$
\begin{aligned}
\left|a_{1} b_{2}-a_{2} b_{1}\right| & =\left|\left[\frac{a_{1}}{h} e_{1}+a_{2} e_{2}\right] \wedge\left[\frac{p_{1}(x)-p_{1}(y)}{h} e_{1}+b_{2} e_{2}\right]\right| h \\
& \leqslant\left(\left|\frac{a_{1}}{h} e_{1}+a_{2} e_{2}\right| \cdot\left|\frac{p_{1}(x)}{h} e_{1}+b_{2} e_{2}\right|+\left|a_{2}\right| \cdot \frac{\left|p_{1}(y)\right|}{h}\right) h \\
& \leqslant\left(.5+.5\left|a_{2}\right|\right) h
\end{aligned}
$$

which we then combine with the relations (11), $a_{3}=0,(13),\left|b_{3}\right| \leqslant .1,\left|a_{2}\right| \leqslant 1$ and $h \geqslant 10^{5}$ to conclude that

$$
\begin{aligned}
\left|v_{1} \wedge v_{2}\right| & =\left[\left(a_{1} b_{2}-a_{2} b_{1}\right)^{2}+\left(a_{1} b_{3}\right)^{2}+\left(a_{2} b_{3}\right)^{2}\right]^{1 / 2} \\
& \leqslant\left[\left(.5+.5\left|a_{2}\right|\right)^{2} h^{2}+.01\left(1-a_{2}^{2}\right) h^{2}+.01\right]^{1 / 2} \\
& =\left[\left(.26+.5\left|a_{2}\right|+.24 a_{2}^{2}\right) h^{2}+.01\right]^{1 / 2} \\
& \leqslant\left(h^{2}+.01\right)^{1 / 2} \leqslant\left(1+10^{-11}\right) h .
\end{aligned}
$$

Case II. $v_{1}, v_{2} \in C-D$ and $a_{2} \geqslant .4$. We infer from the definitions of $M(h), C, D$, that 


$$
\left|a_{1}\right| \leqslant .5\left(1-4 a_{2}^{2}\right)^{1 / 2} h+.5 h \leqslant .8 h,
$$

and then apply this together with the inequalities (11), $\left|b_{2}\right| \leqslant .5,\left|a_{2}\right| \leqslant .5$, $\left|b_{1}\right| \leqslant h,\left|b_{3}\right| \leqslant .1,\left|a_{3}\right| \leqslant .1$ and $h \geqslant 10^{5}$ to obtain

$$
\left|v_{1} \wedge v_{2}\right| \leqslant\left(.8424 h^{2}+.01\right)^{1 / 2} \leqslant h \text {. }
$$

Case III. $v_{1}, v_{2} \in C-D$ and $\left|a_{2}\right| \leqslant .4$. Using the inequalities (11), $\left|a_{1}\right| \leqslant$ $h,\left|b_{2}\right| \leqslant .5,\left|a_{2}\right| \leqslant .4,\left|b_{1}\right| \leqslant h,\left|b_{3}\right| \leqslant .1,\left|a_{3}\right| \leqslant .1$ and $h \geqslant 10^{5}$, we find that

$$
\left|v_{1} \wedge v_{2}\right| \leqslant\left(.85 h^{2}+.0081\right)^{1 / 2} \leqslant h .
$$

Case IV. $v_{1} \in C-D$ and $v_{2} \in D-D$. We combine the relations (11), $b_{2}=0,\left|a_{2}\right| \leqslant .5,\left|b_{1}\right| \leqslant h,\left|a_{1}\right| \leqslant h,\left|b_{3}\right| \leqslant .1,\left|a_{3}\right| \leqslant .1$ and $h \geqslant 10^{5}$ to obtain

$$
\left|v_{1} \wedge v_{2}\right| \leqslant\left(.29 h^{2}+.0025\right)^{1 / 2} \leqslant h .
$$

Case V. $v_{1}, v_{2} \in D-D$. The relations (11), $a_{2}=b_{2}=0,\left|a_{1}\right| \leqslant h,\left|b_{3}\right| \leqslant$ $.1,\left|a_{3}\right| \leqslant .1$ and $\left|b_{1}\right| \leqslant h$ yield $\left|v_{1} \wedge v_{2}\right| \leqslant .2 h$.

4.4. LeMMA. $0<T^{2}(E)<\infty$.

Proof. Since $F_{j}$ is a covering of $A$ by sets of diameter less than $2 \cdot 10^{-24 j}$ for each nonnegative integer $j$, and $\Sigma_{S \in F_{j}} t^{1}(S) \leqslant 2$, it follows that $T^{1}(A) \leqslant 2$. Our conclusion is then obtained by combining this last inequality with Theorem $3.2,[6,2.10 .45]$ and the fact that the ratios are between $T^{m}$ and $H^{m}$ are bounded $[6,2.10 .6]$.

4.5. THEOREM. $T^{2}(E) \leqslant 1-10^{-10}$.

Proof. Choose $\delta>0$ such that

$$
T^{2}(E) \leqslant \sum_{S \in W} t^{2}(S)+10^{-11}
$$

for every countable covering $W$ of $T^{2}$ almost all of $E$ consisting of nonempty subsets of diameter less than $\delta$. Let

$$
\begin{aligned}
\Omega=\{\{x: a \leqslant x<b\} \times T: 0 \leqslant a<b \leqslant 1, \\
\left.T \in \bigcup_{i=0}^{\infty} F_{i}, 10^{5} \operatorname{diam} r_{1}(T) \leqslant b-a \leqslant \delta / 2\right\} .
\end{aligned}
$$

For $S \in \Omega$ let $c(S)$ denote the center of $S$ and then define

$$
\psi(S)=\left\{\left[\operatorname{diam} p_{2}(S)\right] x+c(S): x \in M \frac{\operatorname{diam} p_{1}(S)}{\operatorname{diam} p_{2}(S)}\right\} .
$$

We then choose a sequence $Y_{0}, Y_{1}, Y_{2}, \ldots$ inductively as follows: we let 
$Y_{0}=\varnothing$ and obtain $Y_{n}$ from $Y_{0}, \ldots, Y_{n-1}$ by taking $Y_{n}$ to be a finite disjointed subfamily of $\Omega$ such that

$$
\left(\cup Y_{n}\right) \cap E=\left[E \sim \bigcup_{i=0}^{n-1} \bigcup_{S \in Y_{i}} \psi(S)\right] \sim p_{1}^{-1}\{1\}
$$

Let $Z=\bigcup_{i=0}^{\infty} Y_{i}$. Clearly $\{\psi(S): S \in Z\}$ is a countable disjointed family consisting of nonempty subsets of $E$ of diameter less than $\delta$. Furthermore, we observe that there exists a number $\rho$ such that $0<\rho<1$ and $T^{2}[\psi(S)] / T^{2}(S \cap E)=\rho$ for all $S \in \Omega$, from which it follows that

$$
\begin{aligned}
T^{2}\left[\bigcup_{S \in Y_{n}} \psi(S)\right] & =\rho T^{2}\left[\left(\bigcup Y_{n}\right) \cap E\right] \\
& =\rho T^{2}\left[E \sim \bigcup_{i=0}^{n-1} \bigcup_{S \in Y_{i}} \psi(S)\right]
\end{aligned}
$$

for each positive integer $n$; this in turn implies that

$$
\sum_{n=1}^{j} T^{2}\left[\bigcup_{S \in Y_{n}} \psi(S)\right]=\left[1-(1-\rho)^{j}\right] T^{2}(E)
$$

for all positive integers $j$. Therefore $\{\psi(S): S \in Z\}$ covers $T^{2}$ almost all of $E$ and we may then apply (14) with $W=\{\psi(S): S \in Z\}$ to obtain

$$
T^{2}(E) \leqslant \sum_{S \in Z} t^{2}[\psi(S)]+10^{-11} .
$$

Next we deduce from the definition of $\psi$ and Lemmas 4.2, 4.3 that for all $S \in Z$,

$$
\begin{aligned}
\frac{t^{2}[\psi(S)]}{T^{2}[\psi(S)]} & =\frac{t^{2}\left[M\left(\left[\operatorname{diam} p_{1}(S)\right] /\left[\operatorname{diam} p_{2}(S)\right]\right)\right]}{T^{2}\left[M\left(\left[\operatorname{diam} p_{1}(S)\right] /\left[\operatorname{diam} p_{2}(S)\right]\right)\right]} \\
& \leqslant \frac{1+4 \cdot 10^{-11}}{\left(1+2 \cdot 10^{-10}\right) T^{2}(E)} \leqslant \frac{1-1.5 \cdot 10^{-10}}{T^{2}(E)}
\end{aligned}
$$

Consequently

$$
\sum_{S \in Z} t^{2}[\psi(S)] \leqslant\left(1-1.5 \cdot 10^{-10}\right) \sum_{S \in Z} \frac{T^{2}[\psi(S)]}{T^{2}(E)}=\left(1-1.5 \cdot 10^{-10}\right)
$$

which we then combine with (15) to complete the proof.

5. Final results.

5.1. ThEOREM. $T^{2}(E)<T^{1}(A)$.

Proof. Combine Theorems 3.2, 4.5. 
5.2. Corollary. $C^{2}(E)<C^{1}(A)$.

Proof. $C^{2} \leqslant T^{2}$ by $[6,2.10 .34]$, while clearly $T^{1}=C^{1}$. Therefore

$$
C^{2}(E) \leqslant T^{2}(E)<T^{1}(A)=C^{1}(A) .
$$

5.3. Corollary. $T^{2}(E)<H^{2}(E)$.

Proof. Clearly $T^{1}=H^{1}$, while $H^{1}(S) \leqslant H^{2}([0,1] \times S)$ for all $S \subset \mathbf{R}^{2}$ by $[5,3.6]$. Therefore

$$
T^{2}(E)<T^{1}(A)=H i^{1}(A) \leqslant H^{2}(E) .
$$

\section{REFERENCES}

1. A. S. Besicovitch and P. A. P. Moran, The measure of product and cylinder sets, J. London Math. Soc. 20 (1945), 110-120. MR 8, 18.

2. L. R. Ernst, $A$ proof that $C^{2}$ and $T^{2}$ are distinct measures, Trans. Amer. Math. Soc. 173 (1972), 501-508. MR 46 \#9266.

3. - A proof that $\mathrm{H}^{2}$ and $\mathrm{T}^{2}$ are distinct measures, Trans. Amer. Math. Soc. 191 (1974), 363-372. MR 50 \#13454. 199-202.

4. - $T$ measure of Cartesian product sets, Proc. Amer. Math. Soc. 49 (1975),

5. L. R. Ernst and G. Freilich, A Hausdorff measure inequality, Trans. Amer. Math. Soc. 219 (1976), 361-368. \#1976.

6. H. Federer, Geometric measure theory, Springer-Verlag, New York, 1969. MR 41

7. G. Freilich, On the measure of Cartesian product sets, Trans. Amer. Math. Soc. 69 (1950), 232-275. MR 12, 324.

8. - Carathéodory measure of cylinders, Trans. Amer. Math. Soc. 114 (1965), 384-400. MR 30 \#4892.

9. J. F. Randolph, On generalizations of length and area, Bull. Amer. Math. Soc. 42 (1936), 268-274.

DEPARTMENT OF MATHEMATICS, QUEENS COLLEGE OF THE CITY UNIVERSITY OF NEW YORK, FLUSHING, NEW YORK 11367 